\title{
Photo-leucine and photo-methionine allow identification of protein-protein interactions in living cells
}

\author{
Monika Suchanek, Anna Radzikowska \& Christoph Thiele
}

\begin{abstract}
Protein-protein interactions are the key to organizing cellular processes in space and time. The only direct way to identify such interactions in their cellular environment is by photo-crosslinking. Here we present a new strategy for photo-cross-linking proteins in living cells. We designed two new photoactivatable amino acids that we termed photo-methionine and photo-leucine based on their structures and properties closely resembling the natural amino acids methionine and leucine, respectively. This similarity allows them to escape the stringent identity control mechanisms during protein synthesis and be incorporated into proteins by the unmodified mammalian translation machinery. Activation by ultraviolet light induces covalent cross-linking of the interacting proteins, which can be detected with high specificity by simple western blotting. Applying this technology to membrane protein complexes, we discovered a previously unknown direct interaction of the progesterone-binding membrane protein PGRMC1 with Insig-1, a key regulator of cholesterol homeostasis.
\end{abstract}

Protein-protein interactions are the key to organizing cellular processes in space and time. Recently, systematic efforts ${ }^{1,2}$ have revealed that many, if not most, proteins function in the context of supramolecular complexes rather than as isolated entities. Interest in protein-protein interactions is not confined to basic research. Protein-protein interactions involved in viral fusion or in growthfactor signaling are promising targets for antiviral or anticancer drugs $^{3}$. Such interactions are commonly identified through the use of affinity-based methods such as coimmunoprecipitation. Because these methods use cell lysates as a starting material, the detection of false-positive interactions, resulting from the loss of spatial organization during the lysis procedure, is a problem. In addition, weakly interacting partners can be lost during the wash procedures. Therefore, chemical or photochemical cross-linking has been used to covalently fix interactions in living cells, allowing their detection under stringent conditions. Chemical cross-linking ${ }^{4}$ involves the use of moderately reactive bifunctional reagents, often directed against free amino groups, to generate covalent protein cross-links. Photo-cross-linking ${ }^{5}$, in contrast, generates highly reactive intermediates in situ by irradiation of an inert precursor. The major advantage of photo-cross-linking is greater specificity of crosslinking owing to the short lifetimes of the excited intermediates.

Photo-cross-linking requires the chemical introduction of a photoactivatable group into a protein, which initially limited its scope to small peptides amenable to total chemical synthesis ${ }^{6,7}$. Larger proteins became accessible to modification with the in vitro synthesis of tRNAs bearing a photoactivatable amino acid ${ }^{8,9}$. Introduction of a photoactivatable group into specific positions was achieved using suppressor tRNAs for translation in vitro ${ }^{10}$ and in vivo ${ }^{11,12}$. Using engineered 'orthogonal' pairs of tRNAs and aminoacyl tRNA synthetases (aaRs), various unnatural amino acids now can be incorporated into specific sites of proteins in cultured mammalian cells ${ }^{13-15}$. Whereas this method is well-suited for the production of engineered proteins, its application for global detection of protein-protein interactions in cell biology is limited by the complexity of the modified components (that is, tRNA, aaRS, cDNA and unnatural amino acid) required and by the fact that only a single position in a single protein will bear the photoreactive group.

To study the composition and function of membrane protein complexes that regulate cholesterol homeostasis in the intact living cell, we developed a new strategy. It combines the broad range of chemical cross-linking with the superior specificity of photocross-linking using new photoactivatable amino acids with unique similarity to natural amino acids. These amino acids are efficiently incorporated into proteins by mammalian cells without the need of modified tRNAs or aaRSs, yielding specific crosslinking after photoactivation.

\section{RESULTS}

Synthesis and biosynthetic incorporation into proteins

We synthesized three new amino acids (Fig. 1a and Supplementary Note online) that resemble isoleucine, leucine and methionine in structure but contain a photoactivatable diazirine ring, which yields a reactive carbene after the light-induced loss of nitrogen. All three amino acids were synthesized by $\alpha$-bromination ${ }^{16}$ of the respective azi-carboxylic acids followed by aminolysis of the

Max Planck Institute of Molecular Cell Biology and Genetics, Pfotenhauerstr. 108, D-01307 Dresden, Germany. Correspondence should be addressed to C.T. (thiele@mpi-cbg.de).

PUBLISHED ONLINE 23 MARCH 2005; D0I:10.1038/NMETH752 
a
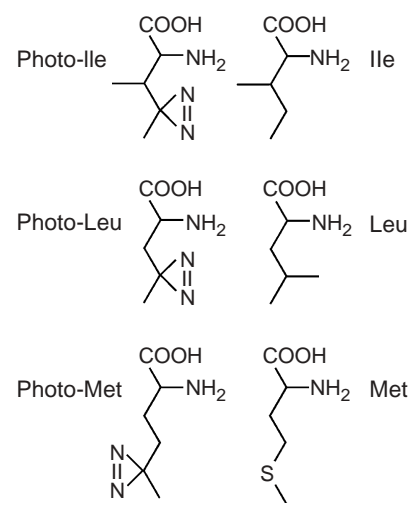

b
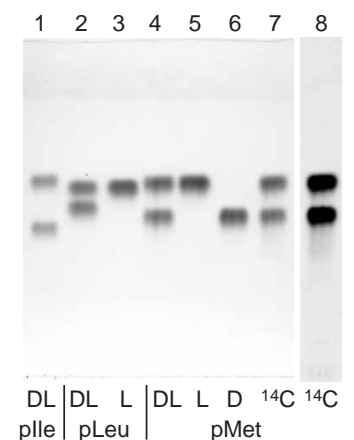

C

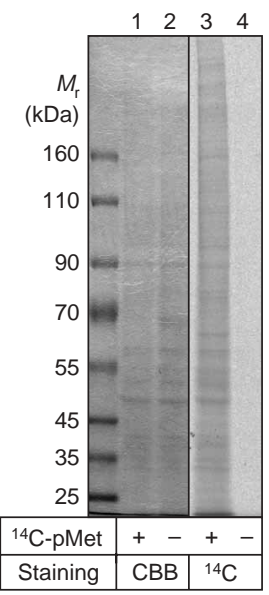

d

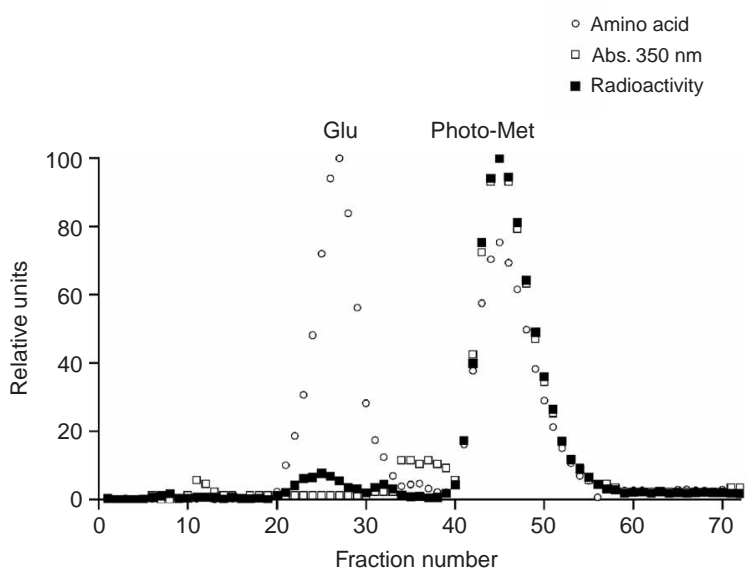

Figure 1 | New photoreactive amino acids are incorporated into proteins. (a) The structures of the three new amino acids, photo-Ile, photo-Leu and photo-Met (left), are shown next to those of the natural amino acids isoleucine, leucine and methionine (right). (b) DL mixtures or pure enantiomers (as indicated) were separated by thin-layer chromatography on a chiral plate. The start and solvent front are at the bottom and top of the image, respectively. Amino acids were detected by ninhydrin staining (lanes 1-7) or autoradiography (lane 8). (c) COS7 cells were grown in the absence (lanes 2 and 4 ) or presence (lanes 1 and 3 ) of $\left[1-{ }^{14} \mathrm{C}\right]$ photo-Met. Proteins were separated by SDS-PAGE and then detected by Coomassie blue staining (lanes 1 and 2) and fluorography (lanes 3 and 4). (d) Precipitated proteins were digested with pronase. An excess of unlabeled glutamine and photo-Met were added, and samples were heated with $7 \mathrm{~N} \mathrm{HCl}$. Amino acids were resolved by chromatography on Dowex 50WX8. Column fractions were assayed for the presence of radioactivity, photo-Met (detected by absorption at $350 \mathrm{~nm}$ ), and amino groups (detected by the fluorescamine assay). Values are corrected for background and normalized to the most intense peak. Total recovery of radioactivity in the column eluate was $85 \%$.

azi-bromo-carboxylic acids. Although photo-Leu and photo-Met were obtained in multigram amounts, 20 -fold less photo-Ile was obtained from the same amount of starting material. In the case of photo-Met, Strecker synthesis ${ }^{17}$ starting from $4,4^{\prime}$-azipentanal also yielded the desired product, but analogous attempts to synthesize photo-Leu and photo-Ile were unsuccessful. Enzymatic resolution of enantiomers gave pure L-photo-Leu, L-photo-Met and D-photoMet (Fig. 1b). We made no attempts to resolve the enantiomers and diastereomers of photo-Ile. DL-photo-Met labeled with ${ }^{14} \mathrm{C}$ was obtained by Strecker synthesis using $\left[{ }^{14} \mathrm{C}\right] \mathrm{NaCN}$ (Fig. 1b).

To demonstrate biosynthetic incorporation of photo-Met into proteins, we cultivated mammalian fibroblast-like COS7 cells in the presence of $\left[1-{ }^{14} \mathrm{C}\right]$ photo-Met. To decrease competition for activation by tRNA synthetases, we used culture medium devoid of the structurally related amino acids leucine and methionine (LM-2) or devoid of leucine, methionine, isoleucine and valine (LM-4). Proteins incorporated the radioactive amino acid, as we were able to detect radioactivity associated with proteins by autoradiography after separation by SDS-PAGE (Fig. 1c, lane 3). To prove that the radioactivity detected represents intact $\left[1-{ }^{14} \mathrm{C}\right]$ photo-Met, we subjected labeled proteins to total hydrolysis and separation of amino acids on an ion-exchange column ${ }^{18}$. Of the radioactivity in the labeled proteins, $88 \%$ was intact $\left[1-{ }^{14} \mathrm{C}\right]$ photo-Met (Fig. 1d, photo-Met). A small peak (7\%) eluting slightly earlier than glutamic acid (Fig. 1d, Glu) was also observed if free $\left[1{ }^{14} \mathrm{C}\right]$ photo-Met was simply heated under the hydrolysis conditions used (data not shown), and we therefore concluded that it represents a thermal decomposition product. Radioactivity originating from degradation followed by reintegration would appear as aspartic acid and glutamic acid, but no radioactivity was incorporated by this pathway. We conclude that at least $95 \%$ of incorporated radioactivity represents intact $\left[1-{ }^{14} \mathrm{C}\right]$ photo-Met. We obtained quantitative data on the frequency of incorporation of photoMet into newly synthesized proteins by colabeling with $\left[1-{ }^{14} \mathrm{C}\right]$ photo-Met and $\left[{ }^{3} \mathrm{H}\right]$ phenylalanine. Comparison of the amount of incorporated isotopes (Table 1) indicated that the frequency of incorporation of $\left[1-{ }^{14} \mathrm{C}\right]$ photo-Met was $14 \%$ of that of $\left[{ }^{3} \mathrm{H}\right] \mathrm{Phe}$, corresponding to $0.7 \%$ of total amino acids.

\section{Photo-amino acids are functional and nontoxic}

Unnatural amino acids are frequently toxic to cells. Therefore, we monitored protein biosynthesis by incorporation of $\left[{ }^{3} \mathrm{H}\right] \mathrm{Phe}$ and determined the viability of cells grown in the presence or absence of photo-amino acids by trypan blue exclusion (Supplementary Table 1 online). Owing to the lack of two essential amino acids, protein biosynthesis was reduced to about $65 \%$ of that in a complete growth medium. Addition of either photo-Leu or photo-Ile did not have any substantial effect, whereas addition of photo-Met increased protein biosynthesis. Although cells grew

Table 1 | Determination of the frequency of incorporation of photo-Met into newly synthesized proteins

\begin{tabular}{lcc}
\hline & {$\left[{ }^{3} \mathrm{H}\right]$ Phe } & {$\left[1-{ }^{14} \mathrm{C}\right]$ photo-Met } \\
\hline Total added (nCi/dish) & 1,000 & 5,000 \\
Specific activity (nCi/nmol) & 4.2 & 4.7 \\
Protein-bound activity (nCi/dish) & $14.5 \pm 1.4$ & $2.34 \pm 0.23$ \\
Incorporated amino acid (nmol/dish) & $3.45 \pm 0.34$ & $0.50 \pm 0.05$ \\
Relative frequency & $4.8 \% 45$ & $0.70 \%$ \\
\hline
\end{tabular}

COS7 cells were grown for $22 \mathrm{~h}$ in LM-2 in the presence of $\left[{ }^{3} \mathrm{H}\right]$ Phe and $\left[1-{ }^{14} \mathrm{C}\right]$ photo-Met. Protein-bound radioactivity was assayed by scintillation counting of protein pellets after chloroform-methanol precipitation. Data are average \pm s.d. of triplicate determinations. 
a

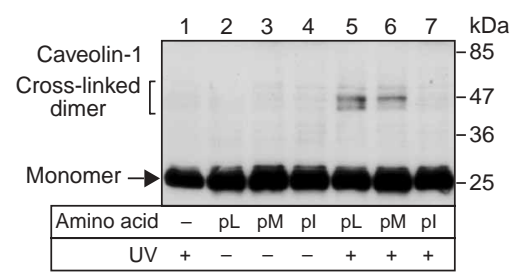

b

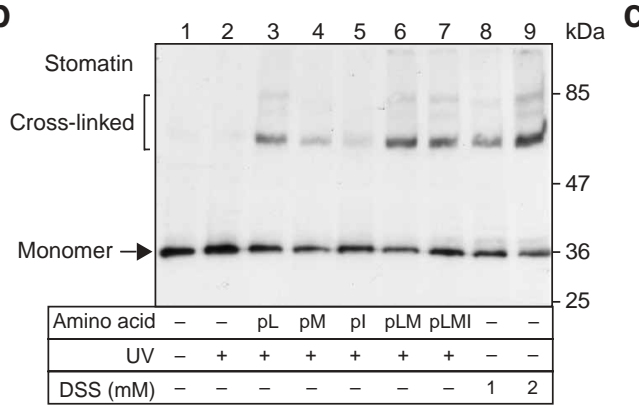

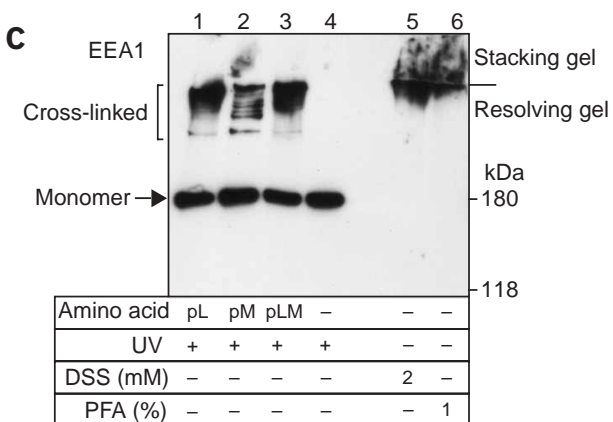

Figure 2 | Photo-cross-linking induced by photo-amino acids. (a-c) COS7 cells $(\mathbf{a}, \mathbf{b})$ or HeLa cells $(\mathbf{c})$ were grown in LM-4 (a) or LM-2 (b,c) in the absence or presence of L-photo-Leu (pL), L-photo-Met (pM) or DL-photo-Ile (pI) or mixtures of these (pLM or pLMI) and then irradiated with UV (+) or not irradiated (-). Cross-linked products formed after UV irradiation were detected by SDS-PAGE and western blotting using anti-caveolin 1 (a) to detect overexpressed caveolin 1 , anti-stomatin (b) to detect endogenous stomatin or anti-EEA1 (c) to detect endogenous EEA1. Cross-linking with two different concentrations of DSS is shown for comparison of cross-linking efficiency (b, lanes 8 and 9). Photo-cross-linking of endogenous EEA1 by photo-Leu and photo-Met as compared to chemical cross-linking using DSS or paraformaldehyde (PFA) as indicated (c).

slightly more slowly than in complete growth medium, their viability (Supplementary Table 1) and morphology (data not shown) were unaffected. Also, photoactivation by UV light for $3 \mathrm{~min}$, sufficient to activate more than $99 \%$ of the activatable groups, did not affect cell viability (Supplementary Table 2 online). We also tested whether proteins produced in cells grown with photo-amino acids are functional or not. To this end, we transfected cells with vectors encoding GFP fusion constructs of the GTPase Rab5 and of constitutively activated (GFPRab5QL) or dominant-negative (GFP-Rab5SN) ${ }^{19}$ mutants, switched cells to media containing photo-amino acids, and monitored the GFP fluorescence by confocal microscopy (Supplementary Fig. 1 online). GFP-Rab5 localized to endosomes, GFP-Rab5QL to enlarged endosomes and GFP-Rab5SN to cytosol, all consistent with previous reports ${ }^{19}$. Both fluorescence intensity and localization were unaffected by the presence of any of the photo-amino acids. At a frequency of substitution of photo-Met for Met of $35 \%$ (see below), more than $80 \%$ of the fusion proteins will have photo-Met in both the Rab5- and the GFP-part of the fusion and more than $99 \%$ in at least one of the two parts. We conclude that photo-amino acids do not impair the function of Rab5 or of GFP.
To corroborate these results with a quantitative assay, we measured the activity of $\beta$-galactosidase that was expressed in the absence or presence of photo-amino acids in the medium (Supplementary Table 3 online). The addition of either of the three photo-amino acids or of a mixture of photo-Met and photo-Leu had no effect on enzyme activity. Escherichia coli $\beta$-galactosidase contains 23 methionines, of which on average 8 would be replaced by photo-Met; less than $0.01 \%$ of the protein is expected to have no substitution by photo-Met. We concluded that photoamino acids are nontoxic to cultivated mammalian cells and can, at least partially, functionally replace branched chain amino acids and methionine.

\section{Specific photo-cross-linking by the 'feed and flash' strategy}

To study photo-cross-linking induced by the photo-amino acids, we transfected COS7 cells with a plasmid containing the gene encoding caveolin-1 (ref. 20), fed the cells with the three photoamino acids and looked for formation of covalent caveolin-1 dimers upon UV irradiation (Fig. 2a). Controls showed that no cross-linking occurred in the absence of photo-amino acids (Fig. 2a, lane 1), and we did not detect any cross-linking in nonirradiated samples in the presence of photo-amino acids a

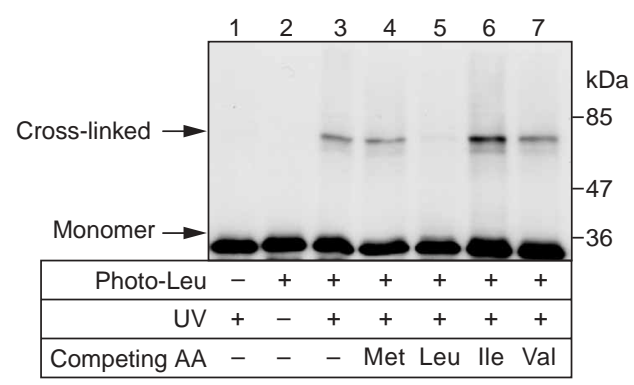

b

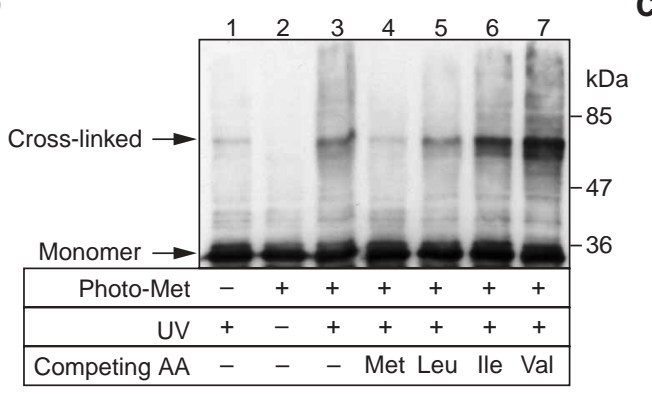

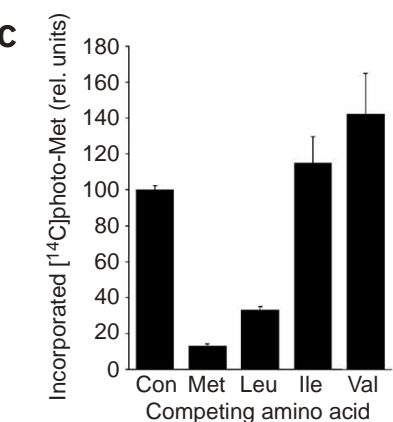

Figure 3 | Specificity of incorporation and cross-linking by photo-Met and photo-Leu. (a,b) COS7 cells transfected with HA-tagged human PGRMC1 were grown in LM-4 in the absence or presence of photo-Leu (a) or photo-Met (b) plus the indicated competing amino acid. Cross-links that formed after UV irradiation were detected by western blotting using antibody to the HA epitope. (c) COS7 cells were grown in the presence of $\left[1-{ }^{14} \mathrm{C}\right]$ photo-Met, a constant trace amount of $\left[{ }^{3} \mathrm{H}\right]$ Phe and competing unlabeled amino acids as indicated. Protein was precipitated and protein-bound radioactivity was determined by scintillation counting. The plot shows the amount of incorporated $\left[1-{ }^{14} \mathrm{C}\right]$ photo-Met, normalized to protein synthesis as determined from incorporation of $\left[{ }^{3} \mathrm{H}\right] \mathrm{Phe}$. The control value obtained in the absence of competing amino acid (Con) is set to 100 . Data are average \pm s.d. of triplicate determinations. 


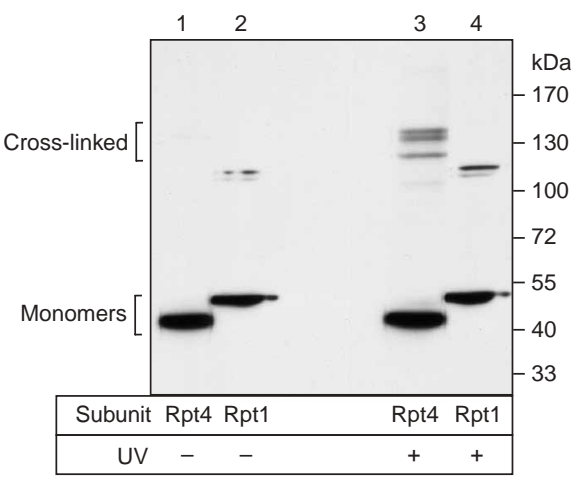

Figure 4 | Photo-cross-linking shows specific interactions of proteasome subunits in vivo. HeLa cells were grown in LM-2 in the presence of photo-Leu and photo-Met and then irradiated with UV (+) or not irradiated (-). Cytosolic fractions were subjected to SDS-PAGE and western blotting against hRpt1 or hRpt4. Rpt4 gives three discrete cross-linked bands, none of which contains Rpt1. A very long exposure shows a cross-linked band of Rpt1 migrating slower than the three cross-linked bands formed by Rpt4 (data not shown).

(Fig. 2a, lanes 2-4). Occasionally, weak cross-linking was induced by irradiation in the absence of photo-amino acids (see an example in Fig. 3b, lane 1), consistent with previous reports on direct UV cross-linking $^{21}$. In samples irradiated in the presence of photoamino acids (Fig. 2a, lanes 5-7), we observed that substantial amounts of a cross-linked dimer were formed with photo-Leu and photo-Met, but not photo-Ile.

Cross-linking with the photo-amino acids was not limited to transfected proteins. The endogenous proteins stomatin ${ }^{22}$ (Fig. 2b) and EEA1 (ref. 23; Fig. 2c) were also cross-linked, with efficiencies comparable to those obtained with the chemical cross-linkers disuccinimidyl suberate (DSS) or paraformaldehyde. For the large protein EEA1 in particular, cross-linking with photo-amino acids was superior to chemical cross-linking because it allowed resolution of discrete cross-linked bands (Fig. 2c, lanes 1-3), whereas chemical cross-linking resulted in formation of highmolecular-weight aggregates that were not resolved by SDS-PAGE (Fig. 2c, lanes 5,6).

The structures of the three photo-amino acids suggest that photo-Ile, photo-Leu and photo-Met might replace isoleucine, leucine and methionine, respectively. If so, addition of the complementary amino acid should strongly reduce incorporation and inhibit photo-cross-linking. We transfected cells with a plasmid encoding the membrane protein PGRMC1 (ref. 24) and assessed whether methionine, valine, leucine or isoleucine competed for cross-linking by photo-Leu and photo-Met. We observed competition for cross-linking by photo-Leu (Fig. 3a, lanes 3-7) mainly from leucine, and competition for cross-linking by photo-Met (Fig. 3b, lanes 3-7) mainly from methionine and to a lesser extent from leucine. Valine and isoleucine did not compete with, but rather enhanced, cross-linking by photo-Met. Methionine and leucine also competed strongly with $\left[1-{ }^{14} \mathrm{C}\right]$ photo-Met for incorporation into proteins, whereas the presence of valine and isoleucine slightly stimulated incorporation of the photo-amino acid (Fig. 3c). Notably, all four of these amino acids are transported into cells by the L-type transport system ${ }^{25}$, which presumably also transports the three photo-amino acids. The strong difference in competition from methionine and leucine as compared to valine and isoleucine excludes the possibility of competition at the level of uptake.

Cross-linking with the photo-amino acids should be very specific, owing to the short half-life of the activated-state carbene and the absence of any spacer molecule. To demonstrate this specificity, we analyzed the cross-linking of subunits of the $19 \mathrm{~S}$ proteasome regulatory particle, a complex of 17 protein subunits. In contrast to the $20 \mathrm{~S}$ core particle of the proteasome, no highresolution structural data for the $19 \mathrm{~S}$ complex are available. Interactions of individual subunits have been mapped by yeast two-hybrid analysis and several other in vitro approaches, resulting in a tentative map of interactions ${ }^{26,27}$. We analyzed the cytosol of photo-cross-linked HeLa cells with antibodies to two of the $19 S$ subunits, Rpt4 (S10b) and Rpt1 (S7) (Fig. 4). After crosslinking, Rpt4 was detected in three distinct cross-linked bands of molecular weights of 120, 140 and $145 \mathrm{kDa}$ (Fig. 4, lane 3), consistent with reported interactions with three other proteasome subunits $^{26}$. In contrast, Rpt1 was not found in any prominent cross-linked band (Fig. 4, lane 4). A band at $115 \mathrm{kDa}$, already detected before irradiation (Fig. 4, lane 2), represented crossreactivity of the antibody. Only a weak band at $155 \mathrm{kDa}$ appeared at much longer exposure time of the blot (data not shown). Despite the high homology of the two proteins (44\% identity, $62 \%$
Figure 5 | Direct interaction in vivo between

Insig- 1 and PGRMC1. (a) COS7 cells were transfected with HA-tagged PGRMC1 and Myctagged Insig- 1 and were grown in LM- 4 in the presence or absence of photo-Met. Cells were UV-irradiated, or not, as indicated, lysed and subjected to immunoprecipitation (IP) with anti$\mathrm{HA}$ or anti-Myc as indicated. Immunoprecipitated proteins were subjected to SDS-PAGE and western blotting (WB) to detect cross-links with anti-Myc or anti-HA as indicated. The small difference in apparent molecular weight of the cross-link is due to different percentages of gels used for SDS-PAGE. The presence of un-cross-linked Insig-1 in the left lanes indicates noncovalent coimmunoprecipitation. The strong band in lanes 3 and 4 (IgG-HC) is cross-reacting IgG heavy chain. (b) COS7 cells were transfected with vectors encoding tagged proteins as indicated, cross-linked and subjected to immunoprecipitation followed by SDS-PAGE and western blotting as above. Note that SCAP forms a high-molecular-weight cross-link, probably a dimer, and Insig-1 cross-links to PGRMC1, whereas Insig-1 does not cross-link to TRP-1-ER, which is also localized to the endoplasmic reticulum.

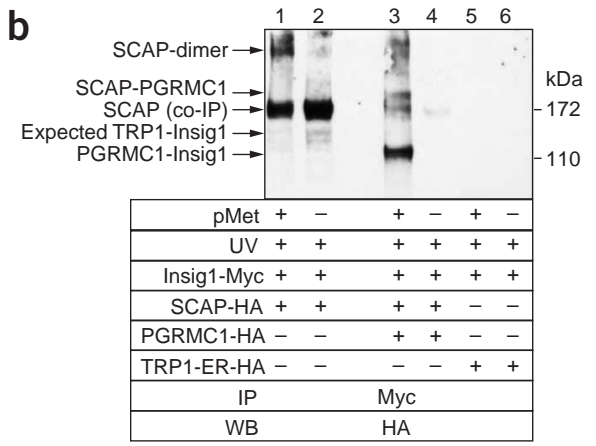

a

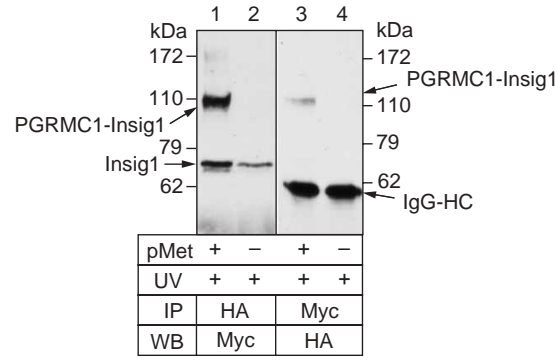
72 0

(1) 
similarity), no common interaction partners were detected, demonstrating the high specificity of the procedure. None of the cross-linked bands that had Rpt 4 contained Rpt1, consistent with the lack of interaction between these two subunits reported in previous studies ${ }^{26,27}$.

\section{PGRMC1 interacts with Insig-1 and SCAP}

We used the new technology to study protein-protein interactions in a complex of membrane proteins involved in the regulation of cellular lipid homeostasis. At least three membrane proteins, SCAP, Insig- 1 and SREBP ${ }^{28-30}$, are part of this assembly, and recent data suggest the existence of additional unidentified components $^{31}$. The complex is located in the endoplasmic reticulum, in which it responds to low cholesterol levels. PGRMC1, a progesterone-binding membrane protein of the endoplasmic reticulum ${ }^{24,32}$, might be another protein involved in this process. In particular, we suspected that it might interact directly with Insig-1. To test this, we coexpressed hemagglutinin (HA)tagged PGRMC1 (PGRMC1-HA) and Myc-tagged Insig-1 (Insig-1-Myc) in COS7 cells, then cultured the cells with or without photo-Met and photo-cross-linked them. To detect the cross-linked proteins, we immunoprecipitated detergent-treated extracts with an antibody to HA and analyzed the precipitates for the presence of Insig-1 by western blotting with an antibody to Myc. When cells were grown in the absence of photo-Met, only a small amount of coimmunoprecipitated Insig-1, but no crosslinked band, was detected (Fig. 5a, lane 2). In cells grown with photo-Met, a strong band appeared at the expected molecular weight of an Insig-1-PGRMC1 cross-linked complex (Fig. 5a, lane 1). We found an identical band using the reverse order of detection, that is, immunoprecipitation with anti-Myc followed by blotting with an antibody to the HA tag (Fig. 5a, lane 3). These results demonstrate a direct interaction between the two proteins in living cells. By the same procedure, we detected a direct interaction between SCAP and PGRMC1 (Fig. 5b, lane 3), but no interaction between Insig-1 and an unrelated overexpressed protein with localization to the endoplasmic reticulum (Fig. 5b, lanes 5 and 6), confirming the specificity of the photocross-linking procedure.

\section{DISCUSSION}

Photo-Met and photo-Leu are readily incorporated into proteins and enable identification of protein-protein interactions upon photoactivation. The basis of this procedure is the unique similarity between the photo-amino acids and the aliphatic hydrophobic amino acids leucine and methionine, which allows the photoamino acids to escape the stringent identity check exerted by the aaRSs. This is interesting fundamentally as well as in the context of the method presented here. Examples of incorporation of unnatural amino acids into proteins by the unmodified endogenous translation machinery are rare $^{33}$ and usually confined to bacteria, and many are based on the particular promiscuity of bacterial MetRS ${ }^{34,35}$. Other aaRSs are more stringent, and replacement of natural amino acids is confined to instances involving isosteric unnatural amino acids such as fluorinated derivatives of leucine, phenylalanine and tryptophan ${ }^{36}$. No example has previously been reported of an unnatural amino acid containing a photoreactive group incorporated into proteins by a eukaryotic cell with genetically unmodified translational machinery.
The frequency of incorporation of photo-amino acids is an important parameter, as quantitative substitution of a natural amino acid will probably yield nonfunctional proteins, whereas a low substitution rate will give poor cross-linking efficiency. The experimentally determined incorporation value for photo-Met of $0.7 \%$ corresponds to a favorable $3-4$ photoactivatable positions in an average 500-aa protein. Given the comparable cross-linking efficiency, we assume photo-Leu to have a similar substitution rate. In some cases-for example, for cross-linking of very small proteins - it might be useful to have a higher degree of substitution. Engineering of editing sites of MetRS and LeuRS, which has been successfully applied to $E$. coli aaRSs ${ }^{37-39}$, should increase the incorporation of photo-Met and photo-Leu, respectively.

Another interesting parameter is the specificity of substitution by photo-Met and photo-Leu. Based on competition by natural amino acids of both incorporation and cross-linking, photo-Leu and photo-Met replace leucine and methionine, respectively. That leucine also weakly competes for photo-Met incorporation might be explained by incorporation of leucine in place of methionine, which would be consistent with the promiscuity reported for bacterial MetRS ${ }^{34,35}$. The preference for methionine and leucine implies that transmembrane domains of membrane proteins, and in general hydrophobic contact areas between proteins ${ }^{40}$, will be preferentially cross-linked; in this the method is complementary to traditional amine-reactive chemical cross-linkers, which target lysine-containing charged regions of proteins. Weak interactions of membrane proteins in the plane of the lipid bilayer, which are difficult to detect by immunoprecipitation in the presence of detergents, can be detected using our cross-linking strategy.

The efficiency of cross-linking that is achieved using the photoamino acids is comparable to that achieved with chemical crosslinkers such as DSS or paraformaldehyde. Its greater specificity makes this method superior to chemical cross-linking, as exemplified by the cross-linking experiments with EEA1 or the proteasome subunit Rpt4. In our hands discrete bands of cross-linked protein are visible on a gel after photo-cross-linking, whereas chemical cross-linking often results in smears on the gel or even unresolvable aggregates. Also, cross-linking with our photo-amino acids did not interfere with recognition of the protein by antibodies, which is a problem with chemical cross-linking directed against the amino group of lysine.

Although designed for the 'feed and flash' strategy, use of our photo-amino acids is by no means restricted to it. Because of the great chemical stability of aliphatic diazirines, all three amino acids can be used in solid-phase peptide synthesis to extend the repertoire of available photo-cross-linking amino acids, which are mostly derivatives of phenylalanine. Engineered incorporation into specific protein positions using modified aaRS-tRNA pairs is another possible application.

To illustrate the power of the new method, we demonstrated a previously undetected protein-protein interaction between PGRMC1 and Insig-1. Both proteins localize to the endoplasmic reticulum $^{29,32}$ : PGRMC1 has been reported to bind progesterone ${ }^{24}$, and Insig-1 is a negative regulator of sterol biosynthesis acting in the SCAP-SREBP system ${ }^{29}$. The physiological implication of this new interaction is under investigation in our laboratory. It should be noted that covalent cross-linking with a zero-distance photo-crosslinker such as photo-Leu or photo-Met is the only way to prove a direct interaction between two proteins of a multiprotein complex 
in the absence of crystallographic data, which are not available for the vast majority of membrane proteins. In general, understanding the organization of functional units in cellular processes will be one of the challenges in postgenomic research. The photo-amino acids described here will allow researchers to determine how the single pieces of the cellular protein puzzle fit together.

\section{METHODS}

Chemical synthesis. For a detailed description of synthetic procedures and characterization of products, see Supplementary Note.

Antibodies. We used mouse monoclonal antibody to HA (clone F-7), rabbit polyclonal antibody to HA (clone Y-11), mouse monoclonal antibody to Myc (clone 9E10), rabbit polyclonal antibody to Myc (clone A-14), rabbit polyclonal antibody to caveolin-1 (clones N-20; all from Santa Cruz), mouse GARP50 antibody to stomatin ${ }^{22}$, mouse monoclonal antibodies p42-23 to human Rpt4 and MSS1-104 to human Rpt1 (both from Biomol), and rabbit polyclonal antiserum to EEA1 (ref. 23).

Expression constructs. A cDNA coding for human caveolin-1 with a C-terminal VSVG tag ${ }^{41}$ was a kind gift of K. Simons. A cDNA for human PGRMC1 (Unigene Hs. 90061) was amplified by PCR from an EST clone (IMAGE 3863377) and was cloned into pcDNA3.1Hygro(+) using HindIII and SpeI restriction sites. Into the resulting construct, a sequence encoding a triple HA tag $(3 \times$ YPYDVPDYA) at the $\mathrm{C}$ terminus of the protein was inserted before the stop codon. SCAP-3HA was constructed as above using IMAGE clone 5271717 and BamHI and SpeI restriction sites of the vector. TRP1-3HA-ER was constructed as above using IMAGE clone 3052338 and EcoRI and XhoI restriction sites of the vector. A sequence encoding an additional endoplasmic reticulum retrieval signal between the $3 \mathrm{HA}$ tag and the $\mathrm{C}$ terminus was added to the construct. A cDNA for human Insig-1 was amplified by PCR from an EST clone (IMAGE 4545505) and was cloned into pcDNA3.1Hygro(+) using HindIII and SpeI restriction sites. Into the resulting construct, a sequence encoding nine successive Myc tags $(9 \times$ EQKLISEEDL) at the $\mathrm{C}$ terminus of the protein was inserted before the stop codon. All products were verified by sequencing. Transfection was performed using Lipofectamine 2000 (Invitrogen) according to the manufacturer's instructions.

Expression and localization to the endoplasmic reticulum of the PGRMC1, SCAP, Insig-1 and TRP-1-ER constructs was verified by western blotting and confocal immunofluorescence microscopy, respectively (data not shown).

Cell culture and labeling with photo-amino acids. COS7 (monkey kidney) cells were grown in high-glucose DMEM (Gibco; with L-glutamine and sodium pyruvate) with $10 \%$ fetal calf serum (FCS). Typically, $0.3 \mathrm{~cm}^{2}$ or $3 \mathrm{~cm}^{2}$ of cells are needed for a crosslinking experiment with detection by western blotting or coimmunoprecipitation and western blotting, respectively. At about $70 \%$ confluence, medium was replaced by LM-4 (DMEM lacking methionine, leucine, isoleucine, valine and phenol red, supplemented with $5 \%$ dialyzed FCS) or by LM-2 (LM-4 with $1 \mathrm{mM}$ of each isoleucine and valine). Photo-amino acids were added to a final concentration of $4 \mathrm{mM}$ photo-Leu and photo-Ile, $1.7 \mathrm{mM}$ photo-Met, and cells were cultivated for $22 \mathrm{~h}$, washed with phosphate-buffered saline (PBS) and UV-irradiated using a
200-W high pressure mercury lamp (Oriel Photomax) with a glass filter to remove wavelengths $<310 \mathrm{~nm}$. The samples were placed at a distance of $38 \mathrm{~cm}$ from the light source, corresponding to an illuminated area of $105-\mathrm{mm}$ diameter. Under these conditions, the $t_{1 / 2}$ for photoactivation, determined following the $350-\mathrm{nm}$ diazirine absorption peak, was $20 \mathrm{~s}$. We irradiated living cells for 1-3 $\mathrm{min}$. Cell viability was unaffected; reduced viability became apparent only at irradiation times much longer than $10 \mathrm{~min}$ (Supplementary Table 2 online). Lysis of cells, SDS-PAGE and western blotting were done as described ${ }^{42}$. For immunoprecipitation, cells were lysed in $20 \mathrm{mM}$ HEPES-NaOH ( $\mathrm{pH}$ 7.4), $100 \mathrm{mM} \mathrm{NaCl}, 5 \mathrm{mM}$ EDTA, 1\% Triton-X 100, 0.5\% sodium deoxycholate, the lysate was cleared with protein A beads and incubated with beads preincubated with polyclonal anti-Myc or anti-HA. Beads were washed three times with $20 \mathrm{mM}$ HEPES$\mathrm{NaOH}(\mathrm{pH} 7.25), 0.5 \%$ CHAPS and with $100 \mathrm{mM}$ Tris- $\mathrm{HCl}$ (pH 8.3), $150 \mathrm{mM} \mathrm{NaCl}, 2 \mathrm{mM}$ EDTA, 0.1\% (wt/vol) SDS, $0.5 \%$ (wt/vol) Nonidet P-40, 0.5\% (wt/vol) sodium deoxycholate. Proteins were eluted by heating with reducing SDS-PAGE sample buffer.

For the experiment shown in Figure 4, HeLa cells were grown in LM-2 with $1.7 \mathrm{mM}$ photo-Met and $4 \mathrm{mM}$ photo-Leu, and UV irradiated or not. Cells were washed with PBS and HB (15 mM HEPES-NaOH (pH 7.4) with $0.25 \mathrm{M}$ sucrose), scraped into HB with protease inhibitors and homogenized in an EMBL cell cracker (on ice, 12- $\mu \mathrm{M}$ clearance, 10 strokes). Homogenates were centrifuged at $3,000 \mathrm{~g}$ for $5 \mathrm{~min}$ and the supernatants were centrifuged at $60,000 \mathrm{~g}$ for $1 \mathrm{~h}$ at $4{ }^{\circ} \mathrm{C}$. Proteins in the final supernatants were precipitated with chloroform-methanol and subjected to SDSPAGE and western blotting.

Chemical cross-linking. COS7 or HeLa cells were grown in LM-2, washed with PBS, incubated with 1-2 mM of DSS or with $1 \%$ paraformaldehyde in PBS for $30 \mathrm{~min}$ at room temperature, and finally quenched for $15 \mathrm{~min}$ with $10 \mathrm{mM}$ Tris ( $\mathrm{pH} \mathrm{8.6)} \mathrm{or}$ $5 \mathrm{mM}$ glycine, respectively. Cell lysis and processing for SDS-PAGE and western blotting was done as described above.

Analysis of incorporation of radioactive amino acids into protein. COS7 cells were grown for $20 \mathrm{~h}$ in LM-2 plus $\left[1-{ }^{14} \mathrm{C}\right]$ photo-Met and $\left[{ }^{3} \mathrm{H}\right] \mathrm{Phe}$, washed $6 \times$ with $\mathrm{PBS}$ and lysed with $0.5 \%$ Triton $\mathrm{X}-100,0.2 \%$ sodium deoxycholate. Aggregated DNA was removed, protein was precipitated $^{43}$, the pellet was redissolved in $0.3 \mathrm{ml}$ $0.5 \%$ SDS and the precipitation was repeated. More than $99 \%$ of total radioactivity precipitated, demonstrating complete removal of free amino acids. The pellet was redissolved and processed for scintillation counting (data in Table $\mathbf{1}$ and Fig. 3c) or processed as follows for amino acid analysis (Fig. 1d): the pellet was dissolved in $600 \mu \mathrm{l} 50 \mathrm{mM} \mathrm{NH}_{4} \mathrm{HCO}_{3}, 0.5 \%$ SDS, $3 \mathrm{mM} \mathrm{CaCl}_{2}$ containing $0.25 \mathrm{mg}$ pronase (Roche) and incubated at $37{ }^{\circ} \mathrm{C}$ for $20 \mathrm{~h}$. The sample was lyophilized, dissolved in $600 \mu \mathrm{l}$ of $7 \mathrm{~N} \mathrm{HCl}$ containing $4 \mathrm{mg}$ of glutamic acid and $6 \mathrm{mg}$ of photo-Met and heated to $70{ }^{\circ} \mathrm{C}$ for $24 \mathrm{~h}$. A Dowex 50WX8 column ${ }^{18}(1.6 \times 24 \mathrm{~cm})$ equilibrated with $0.1 \mathrm{M}$ sodium citrate ( $\mathrm{pH}$ 3.8). The sample was lyophilized, dissolved in $1 \mathrm{ml} 0.1 \mathrm{M}$ sodium citrate ( $\mathrm{pH} 3.8$ ) and loaded onto the column. Proteins were eluted from the column at $24{ }^{\circ} \mathrm{C}$ at $20 \mathrm{ml} / \mathrm{h}$ with the following volumes of $0.1 \mathrm{M}$ sodium citrate buffers: $70 \mathrm{ml}(\mathrm{pH} \mathrm{3.8),} 26 \mathrm{ml}(\mathrm{pH} 4.3)$ and $100 \mathrm{ml}(\mathrm{pH} \mathrm{4.5)}$. Fractions of $3 \mathrm{ml}$ each were collected and assayed for absorbance 
at $350 \mathrm{~nm}$, radioactivity was measured by scintillation counting, and the presence of amino groups was assayed by the fluorescamine method ${ }^{44}$.

Note: Supplementary information is available on the Nature Methods website.

\section{ACKNOWLEDGMENTS}

We are grateful to J. Rink for help with Rab experiments, M. Zerial for the GFP-Rab expression constructs and antibody against EEA1, R. Prohaska for antibody against Stomatin, F. Buchholz for the $\beta$-galactosidase expression vector, A. Rudolph and M. Gruner for recording of NMR spectra, G. Wiebe for DNA sequencing and K. Simons and T. Kurzchalia for critical reading of the manuscript.

\section{COMPETING INTERESTS STATEMENT}

The authors declare competing financial interests (see the Nature Methods website for details).

\section{Received 30 November 2004; accepted 28 February 2005}

Published online at http://www.nature.com/naturemethods/

1. Ho, Y. et al. Systematic identification of protein complexes in Saccharomyces cerevisiae by mass spectrometry. Nature 415, 180-183 (2002).

2. Gavin, A.C. et al. Functional organization of the yeast proteome by systematic analysis of protein complexes. Nature 415, 141-147 (2002).

3. Gadek, T.R. Strategies and methods in the identification of antagonists of protein-protein interactions. Biotechniques (Suppl.) 34, 21-24 (2003).

4. Ji, T.H. Bifunctional reagents. Methods Enzymol. 91, 580-609 (1983).

5. Bayley, H. \& Knowles, J.R. Photoaffinity labeling. Methods Enzymol. 46, 69-114 (1977).

6. Jacobs, S., Hazum, E., Shechter, Y. \& Cuatrecasas, P. Insulin receptor: covalent labeling and identification of subunits. Proc. Natl. Acad. Sci. USA 76, 4918-4921 (1979).

7. Thiele, C. \& Fahrenholz, F. Photoaffinity labeling of central cholecystokinin receptors with high efficiency. Biochemistry 32, 2741-2746 (1993).

8. Kurzchalia, T.V. et al. The signal sequence of nascent preprolactin interacts with the 54K polypeptide of the signal recognition particle. Nature 320, 634-636 (1986).

9. Krieg, U.C., Walter, P. \& Johnson, A.E. Photocrosslinking of the signal sequence of nascent preprolactin to the 54-kilodalton polypeptide of the signal recognition particle. Proc. Natl. Acad. Sci. USA 83, 8604-8608 (1986).

10. Cornish, V.W. et al. Site-specific incorporation of biophysical probes into proteins. Proc. Natl. Acad. Sci. USA 91, 2910-2915 (1994).

11. Chin, J.W., Martin, A.B., King, D.S., Wang, L. \& Schultz, P.G. Addition of a photocrosslinking amino acid to the genetic code of Escherichia coli. Proc. Natl. Acad. Sci. USA 99, 11020-11024 (2002).

12. Deiters, A. et al. Adding amino acids with novel reactivity to the genetic code of Saccharomyces cerevisiae. J. Am. Chem. Soc. 125, 11782-11783 (2003).

13. Sakamoto, K. et al. Site-specific incorporation of an unnatural amino acid into proteins in mammalian cells. Nucleic Acids Res. 30, 4692-4699 (2002).

14. Monahan, S.L., Lester, H.A. \& Dougherty, D.A. Site-specific incorporation of unnatural amino acids into receptors expressed in mammalian cells. Chem. Biol. 10, 573-580 (2003).

15. Zhang, Z. et al. Selective incorporation of 5-hydroxytryptophan into proteins in mammalian cells. Proc. Natl. Acad. Sci. USA 101, 8882-8887 (2004).

16. Harrp, D.N., Bao, L.Q., Black, C.J., Gleason, J.G. \& Smith, R.A. An efficient $\alpha$-halogenation of acyl chlorides by $N$-bromosuccinimide, $N$-chlorosuccinimide, and molecular iodine. J. Org. Chem. 40, 3420-3427 (1975).

17. Strecker, A. Ueber die kuenstliche Bildung der Milchsaeure und eines neuen, dem Glycocoll homologen Koerper. Liebigs Ann. Chem. 75, 27-45 (1850).

18. Moore, S. \& Stein, W.H. Chromatography of amino acids on sulfonated polystyrene resins. J. Biol. Chem. 192, 663-681 (1951).

19. Stenmark, H. et al. Inhibition of rab5 GTPase activity stimulates membrane fusion in endocytosis. EMBO J. 13, 1287-1296 (1994).

20. Sargiacomo, M. et al. Oligomeric structure of caveolin: implications for caveolae membrane organization. Proc. Natl. Acad. Sci. USA 92, 9407-9411 (1995).
21. Thibonnier, M. The human platelet vasopressin receptor identification by direct ultraviolet photoaffinity labeling. J. Biol. Chem. 262, 10960-10964 (1987).

22. Snyers, L., Umlauf, E. \& Prohaska, R. Oligomeric nature of the integral membrane protein stomatin. J. Biol. Chem. 273, 17221-17226 (1998).

23. Simonsen, A. et al. EEA1 links PI(3)K function to Rab5 regulation of endosome fusion. Nature 394, 494-498 (1998).

24. Falkenstein, E., Meyer, C., Eisen, C., Scriba, P.C. \& Wehling, M. Full-length cDNA sequence of a progesterone membrane-binding protein from porcine vascular smooth muscle cells. Biochem. Biophys. Res. Commun. 229, 86-89 (1996).

25. Kanai, Y. et al. Expression cloning and characterization of a transporter for large neutral amino acids activated by the heavy chain of 4F2 antigen (CD98). J. Biol. Chem. 273, 23629-23632 (1998).

26. Ferrell, K., Wilkinson, C.R., Dubiel, W. \& Gordon, C. Regulatory subunit interactions of the $26 \mathrm{~S}$ proteasome, a complex problem. Trends Biochem. Sci. 25, 83-88 (2000).

27. Fu, H., Reis, N., Lee, Y., Glickman, M.H. \& Vierstra, R.D. Subunit interaction maps for the regulatory particle of the $26 \mathrm{~S}$ proteasome and the COP9 signalosome. EMBO J. 20, 7096-7107 (2001).

28. Brown, M.S. \& Goldstein, J.L. A proteolytic pathway that controls the cholesterol content of membranes, cells, and blood. Proc. Natl. Acad. Sci. USA 96, 11041-11048 (1999).

29. Yang, T. et al. Crucial step in cholesterol homeostasis: sterols promote binding of SCAP to INSIG-1, a membrane protein that facilitates retention of SREBPS in ER. Cell 110, 489-500 (2002).

30. Horton, J.D., Goldstein, J.L. \& Brown, M.S. SREBPs: transcriptional mediators of lipid homeostasis. Cold Spring Harb. Symp. Quant. Biol. 67, 491-498 (2002).

31. Adams, C.M. et al. Cholesterol and 25-hydroxycholesterol inhibit activation of SREBPs by different mechanisms, both involving SCAP and insigs. J. Biol. Chem. 279, 52772-52780 (2004).

32. Nolte, I., Jeckel, D., Wieland, F.T. \& Sohn, K. Localization and topology of ratp28, a member of a novel family of putative steroid-binding proteins. Biochim. Biophys. Acta 1543, 123-130 (2000).

33. Hendrickson, T.L., de Crecy-Lagard, V. \& Schimmel, P. Incorporation of nonnatural amino acids into proteins. Annu. Rev. Biochem. 73, 147-176 (2004).

34. Kiick, K.L., Weberskirch, R. \& Tirrell, D.A. Identification of an expanded set of translationally active methionine analogues in Escherichia coli. FEBS Lett. 502, 25-30 (2001).

35. Link, A.J., Mock, M.L. \& Tirrell, D.A. Non-canonical amino acids in protein engineering. Curr. Opin. Biotechnol. 14, 603-609 (2003).

36. Rennert, 0.M. \& Anker, H.S. On the incorporation of $5^{\prime}, 5^{\prime}, 5^{\prime}$-trifluoroleucine into proteins of E. coli. Biochemistry 13, 471-476 (1963).

37. Doring, V. et al. Enlarging the amino acid set of Escherichia coli by infiltration of the valine coding pathway. Science 292, 501-504 (2001).

38. Mursinna, R.S. \& Martinis, S.A. Rational design to block amino acid editing of a tRNA synthetase. J. Am. Chem. Soc. 124, 7286-7287 (2002).

39. Tang, Y. \& Tirrell, D.A. Attenuation of the editing activity of the Escherichia coli leucyl-tRNA synthetase allows incorporation of novel amino acids into proteins in vivo. Biochemistry 41, 10635-10645 (2002).

40. Jones, S. \& Thornton, J.M. Principles of protein-protein interactions. Proc. Natl. Acad. Sci. USA 93, 13-20 (1996).

41. Dupree, P., Parton, R.G., Raposo, G., Kurzchalia, T.V. \& Simons, K. Caveolae and sorting in the trans-Golgi network of epithelial cells. EMBO J. 12, 1597-1605 (1993).

42. Thiele, C., Hannah, M.J., Fahrenholz, F. \& Huttner, W.B. Cholesterol binds to synaptophysin and is required for biogenesis of synaptic vesicles. Nat. Cell Biol. 2, 42-49 (2000)

43. Wessel, D. \& Fluegge, U.I. A method for the quantitative recovery of protein in dilute solution in the presence of detergents and lipids. Anal. Biochem. 138, 141-143 (1984).

44. Udenfriend, S. et al. Fluorescamine: a reagent for assay of amino acids, peptides, proteins, and primary amines in the picomole range. Science $178,871-872$ (1972).

45. Tourasse, N.J. \& Li, W.H. Selective constraints, amino acid composition, and the rate of protein evolution. Mol. Biol. Evol. 17, 656-664 (2000). 